Сравнение результатов у пациентов с Normal Flow High Gradient и Low Flow Low Gradient после коррекции выраженного аортального стеноза процедурой Ozaki в отдаленном периоде наблюдения

\author{
В.В. Базылев ${ }^{\bowtie}$, Д.С. Тунгусов, Р.М. Бабуков, Ф.Л. Бартош, А.И. Микуляк, А.В. Горшкова \\ ФГБУ «Федеральный центр сердечно-сосудистой хирургии» Минздрава России, Пенза, Россия \\ cardio-penza@yandex.ru
}

\begin{abstract}
Аннотация
Актуалъностъ. В нескольких крупных исследованиях было доказано, что пациенты с Low Flow Low Gradient (LFLG) после протезирования аортального клапана (АК) биологическими или механическими протезами имеют более высокую смертность и количество неблагоприятных событий по сравнению с пациентами с Normal Flow High Gradient (NFHG). Oднако на сегодняшний день нет сравнительных исследований пациентов этих групп после процедуры протезирования створок аутоперикардом по методике Ozaki. Актуальность исследования заключается в том, что значительно лучшие гемодинамические свойства створок из аутоперикарда по сравнению с биологическими протезами могут несколько благоприятнее отражаться на результатах у пациентов с LFLG в ближайших и отдаленных периодах наблюдения.

цель. 1. Сравнить ближайшие и отдаленные результаты пациентов группы LFLG с результатами пациентов группы NFHG после реконструкции АК аутоперикардом по методике Ozaki. 2. Определить предикторы госпитальной и отдаленной летальности у пациентов с LFLG.

Материалы илмтоды. Все исследуемые пациенты были разделены на две группы: 1-я - 137 пациентов с NFHG с признаками классического аортального стеноза AVA $<1 \mathrm{~cm}^{2}, \mathrm{Gmean}>40$ мм рт. ст., индексом ударного объема левого желудоч-

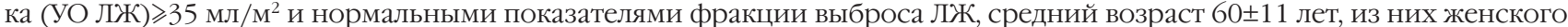
пола - 20 пациентов; 2-я - 71 пациент с LFLG - пациенты, у которых на фоне снижения индекса УО лж<35 мл/м² или систолической функции ЛЖ отмечалась недооценка показателей среднего градиента Gmean<40 мм рт. ст., несмотря на незначительную площадь отверстия AК AVA $<1 \mathrm{~cm}^{2}$. Средний возраст $61 \pm 10$ лет, из них женского пола - 43 пациента.

Peзультатъ. Госпитальная летальность после хирургической коррекции АК была достоверно выше у пациентов в группе с LFLG, чем у пациентов в группе с NFHG, 3 (4,2\%) пациента - 1 (0,7\%) пациент соответственно $(p=0,002)$. Выживаемость в максимальный период наблюдения для пациентов с LFLG была значительно ниже, чем в группе пациентов с NFHG: 88,6 (95\% доверительный интервал - ДИ 44-49,6) и 97,8 (95\% ДИ 48,9-51) соответственно ( $p=0,009)$. По результатам регрессии Кокса независимыми предикторами летальности в отдаленном периоде наблюдения у пациентов с LFLG являются и ударный объем [отношение шансов 0,8 (95\% Ди 0,9-1,1); $p=0,008$ ] и глобальная продольная деформация Лж [отношение шансов 0,56 (95\% ДИ 0,47-1,1); $p=0,01$ ].

Выводы. 1. После процедуры Ozaki пациенты группы с LFLG имеют более высокие риски неблагоприятных событий как на госпитальном этапе, так и в отдаленном периоде наблюдения по сравнению с пациентами с NFHG. 2. Предикторами госпитальной летальности в группе пациентов c LFLG являются длительность ишемии и масса ЛЖ. 3. Предикторами летальности в отдаленном периоде у пациентов с LFLG являются индекс УО ЛЖ и глобальная продольная деформация ЛЖ.

Ключевые слова: Low Flow Low Gradient, операция Ozaki, аортальный стеноз, индекс ударного объема, глобальная продольная деформация левого желудочка.

Для цитирования: Базылев В.В., Тунгусов Д.С., Бабуков, Р.М. и др. Сравнение результатов у пациентов с Normal Flow High Gradient и Low Flow Low Gradient после коррекции выраженного аортального стеноза процедурой Оzaki в отдаленном периоде наблюдения. CardioСоматика. 2020; 11 (2): 25-32. DOI: 10.26442/22217185.2020.2.200098
\end{abstract}

Original article

\title{
Comparison of the results in Normal Flow High Gradient and Low Flow Low Gradient patients after correction of pronounced aortic stenosis with Ozaki procedure in the long-term observation
}

\footnotetext{
Vladlen V. Bazylev ${ }^{\bowtie}$, Dmitrii S. Tungusov, Ruslan M. Babukov, Fedor L. Bartosh, Artur I. Mikulyak, Alena V. Gorshkova

Federal Center of Cardiovascular Surgery, Penza

cardio-penza@yandex.ru
}

\section{Abstract}

Relevance. It has been proven that patients with Low Flow Low Gradient (LFLG) after aortic valve replacement with biological or mechanical prostheses have a higher mortality rate and the number of adverse events compared with patients with Normal 
Flow High Gradient (NFHG). However, there are currently no comparative studies of patients with NFHG and LFLG after the Ozaki procedure. The better hemodynamic properties of autopericardial cusps compared with biological prostheses can more favorably influence the results in patients with LFLG in the short and long-term follow-up periods.

Aim. 1. Compare the hospit and long-term results of patients of the LFLG group with the results of patients of the NFHG group after the Ozaki procedure. 2. Identify predictors of hospital and long-term mortality in patients with LFLG.

Materials and methods. All patients have been divided into two groups. Group 1: 137 patients with NFHG and signs of classic aortic stenosis: $\mathrm{AVA}<1 \mathrm{~cm}^{2}$, Gmean $>40, \mathrm{SV}$ index $\geqslant 35 \mathrm{ml} / \mathrm{m}^{2}$ and normal left ventricle (LV) ejection fraction. Group 2.71 patients with LFLG and underestimation of the average gradient indices (Gmean<40) despite a decrease in the aortic valve aperture $\mathrm{AVA}<1 \mathrm{~cm}^{2}$ amid a decrease in the index of stroke volume $<35 \mathrm{ml} / \mathrm{m}^{2}$ and $\mathrm{LV}$ systolic function.

Results. Hospital mortality after surgical correction of AV stenosis was significantly higher in patients of group 2: 3 (4.2\%) patients and $1(0.7 \%)$ patients, respectively $(p=0.002)$. Survival at the maximum follow-up period for patients with LFLG was significantly lower than in the group of patients with NFHG 88.6 (95\% confidence interval - CI 44-49.6) and 97.8 (95\% CI 48.9-51), respectively $(p=0.009)$. According to the results of the Cox regression analysis, the independent predictors of mortality in the longterm follow-up of patients with LFLG are the SV odds ratio 0.8 (95\% CI 0.9-1.1); $p=0.008$ and the global longitudinal LV deformation (GLS) odds ratio 0.56 (95\% CI 0.47-1.1); $p=0.01$.

Conclusions. 1. After the Ozaki procedure, patients with the LFLG group have higher risks of adverse events, both at the hospital stage and in the long-term follow-up, compared to patients with NFHG. 2. The duration of ischemia and LV mass are predictors of hospital mortality in the LFLG group of patients. 3. Predictors of long-term mortality in patients with LFLG are LV stroke volume index and global longitudinal LV deformation.

Key words. Low Flow Low Gradient, Ozaki surgery, aortic stenosis, stroke volume index, global longitudinal deformity of the left ventricle.

For citation: Bazylev V.V., Tungusov D.S., Babukov R.M. et al. Comparison of the results in Normal Flow High Gradient and Low Flow Low Gradient patients after correction of pronounced aortic stenosis with Ozaki procedure in the long-term observation. Cardiosomatics. 2020; 11 (2): 25-32. DOI: 10.26442/22217185.2020.2.200098

\section{Введение}

Аортальный стеноз (АС) является наиболее распространенным заболеванием клапанного аппарата и наиболее частым показанием для замены аортального клапана (АК) у взрослых. Согласно современным рекомендациям, стеноз АК принято считать выраженным, если площадь эффективного отверстия АК $(\mathrm{AVA}) \leqslant 1 \quad \mathrm{~cm}^{2}$, индекс площади отверстия (iAVA) $\leqslant 0,6 \mathrm{~cm}^{2} / \mathrm{M}^{2}$, трансаортальный средний градиент (Gmean) $\geqslant 40$ мм рт. ст. или пиковая скорость $\geqslant 4$ м/с и пиковый градиент $\geqslant 64$ мм рт. ст. [1]. Данную группу пациентов, согласно современным рекомендациям, принято обозначать как пациентов с нормальным потоком и высоким трансаортальным градиентом Normal Flow High Gradient (NFHG) [2]. Однако на фоне сниженной фракции выброса (ФВ) левого желудочка (ЛЖ) $<50 \%$, сниженного сердечного выброса и индексированного ударного объема (УО) Лж<35мл/м² могут возникать расхождения между показателями AVA и средним градиентом на АK (Gmean), т.е. значение Gmean может быть низким (<40 мм рт. ст.), несмотря на небольшую вычисленную площадь отверстия AK AVA $\left(\leqslant 1 \mathrm{~cm}^{2}\right)$. Таких пациентов относят к группе АС с низким потоком и низким трансаортальным градиентом - Low Flow Low Gradient (LFLG) [2]. В нескольких крупных исследованиях было доказано, что пациенты с LFLG после протезирования АК биологическими или механическими протезами имеют более высокие смертность и количество неблагоприятных событий по сравнению с пациентами с NFHG [3-8]. Одни исследователи связывают это с повышенными рисками пациент-протезного несоответствия, другие - с более выраженным нарушением геометрии и функции миокарда [9-13].

Метод реконструкции створок аутоперикардом обладает лучшей гемодинамикой АК по сравнению с биологическими протезами и ограничивает пациента от осложнений, связанных с пациент-протезным несоответствием [14]. Мы предположили, что значительно лучшие гемодинамические свойства створок из аутоперикарда, близких к нативному клапану, и отсутствие осложнений, связанных с пациент-протезным несоответствием, могут несколько благоприятнее отражаться на результатах у пациентов с LFLG в ближайших и отдаленных периодах наблюдения.

\section{Цели исследования:}

1. Сравнить ближайшие и отдаленные результаты пациентов групп с LFLG с результатами пациентов группы с NFHG после реконструкции АК аутоперикардом по методике Ozaki.

2. Определить предикторы госпитальной и отдаленной летальности у пациентов с LFLG.

\section{Материалы и методы}

За текущий период с 2015 г. по декабрь 2019 г. выполнено 654 реконструкции створок аутоперикардом по методике Ozaki. Исходные характеристики, процедурные данные и результаты были собраны проспективно и сохранены в электронной базе данных в рамках исследования по изучению отдаленных результатов процедуры протезирования створок аутоперикардом по методике Ozaki. Исследование было одобрено местным этическим комитетом, письменное информированное согласие было получено у всех пациентов. Из анализа исключены пациенты с сопутствующей хирургической процедурой на другом клапане, сопутствующей хирургической процедурой на коронарных артериях и пациенты, которые были оперированы по поводу умеренной или выраженной недостаточности на АК. Сбор данных проводился на госпитальном этапе, в период 6-12 мес после оперативного вмешательства с последующим ежегодным осмотром и регистрацией данных до 4 лет. Пациентам, которые не могли по каким-либо причинам прибыть в клинику для осмотра, проводили опрос по телефону, чтобы определить выживаемость и функциональный класс (ФК) NYHA. Данные эхокардиографии этих пациентов были предоставлены по электронной почте.

Все исследуемые были разделены на две группы:

- 1-я группа - 137 пациентов с NFHG с признаками классического AC, AVA $<1 \mathrm{~cm}^{2}$, Gmean $>40$ мм рт. ст., индексом УО ЛЖ $\geqslant 35$ мл/м² и нормальными показа-

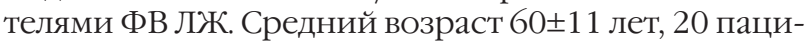
ентов женского пола.

- 2-я группа - 71 пациент с LFLG - пациенты, у кото-

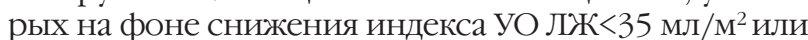
систолической функции ЛЖ отмечалась недооценка показателей среднего градиента Gmean<40 мм рт. ст., 


\begin{tabular}{|c|c|c|c|}
\hline Показатели & NFHG $(n=137)$ & LFLG (n=71) & $p$ \\
\hline Индекс массы тела, кг/м² & $27,8 \pm 5,8$ & $29,4 \pm 5,0$ & 0,08 \\
\hline Площадь поверхности тела, м² & $1,88 \pm 0,2$ & $1,9 \pm 0,2$ & 0,2 \\
\hline Сахарный диабет & $32(12 \%)$ & $8(12 \%)$ & 0,9 \\
\hline Euro SCORE I & $5,0 \pm 0,8$ & $6,9 \pm 2$ & 0,002 \\
\hline Euro SCORE II & $2,8 \pm 0,4$ & $3,9 \pm 0,9$ & 0,008 \\
\hline Артериальная гипертензия & $130(80 \%)$ & $68(95 \%)$ & 0,03 \\
\hline Мультифокальный атеросклероз & $20(14 \%)$ & $9(13 \%)$ & 0,1 \\
\hline Нарушение функции почек & $9(6,5 \%)$ & $7(9,8 \%)$ & 0,08 \\
\hline Хроническая обструктивная болезнь легких & $19(13,8 \%)$ & $3(4 \%)$ & 0,009 \\
\hline ФП & $12(9 \%)$ & $9(12,6 \%)$ & 0,008 \\
\hline ФKI (NYHA) & $16(12 \%)$ & $6(9 \%)$ & 0,03 \\
\hline ФK II (NYHA) & $59(43 \%)$ & $9(14 \%)$ & $<0,001$ \\
\hline ФK III (NYHA) & $61(44,5 \%)$ & $45(69 \%)$ & $<0,001$ \\
\hline ФK IV (NYHA) & $1(0,5 \%)$ & $5(7 \%)$ & $<0,001$ \\
\hline Средний ФК (NYHA) & $2,0 \pm 1,3$ & $2,6 \pm 1,1$ & 0,007 \\
\hline 6-минутный тест, м & $347 \pm 90$ & $276 \pm 84$ & $<0,001$ \\
\hline КдО лж, мл & $140 \pm 30$ & $134 \pm 30$ & 0,06 \\
\hline ФВ лЖ, \% & $64 \pm 13$ & $47 \pm 7$ & $<0,001$ \\
\hline Эффективный индекс УО ЛЖ, мл/м² & $38 \pm 3$ & $29 \pm 7$ & $<0,001$ \\
\hline GLS ЛЖ, \% & $13 \pm 4$ & $10 \pm 3$ & $<0,001$ \\
\hline Индекс объема ЛП & $45 \pm 7$ & $50 \pm 10$ & 0,008 \\
\hline Индекс массы ЛЖ, г/м² & $178 \pm 20$ & $175 \pm 21$ & 0,1 \\
\hline Gmax, мм рт. ст. & $85 \pm 21$ & $55 \pm 9$ & $<0,001$ \\
\hline Gmean, мм рт. ст. & $50 \pm 17$ & $31,8 \pm 8$ & $<0,001$ \\
\hline AVA, $\mathrm{CM}^{2}$ & $0,9 \pm 0,2$ & $0,67 \pm 0,1$ & $<0,001$ \\
\hline iAVA, $\mathrm{CM}^{2} / \mathrm{M}^{2}$ & $0,47 \pm 0,01$ & $0,35 \pm 0,1$ & $<0,001$ \\
\hline DVI & $0,21 \pm 0,3$ & $0,20 \pm 0,3$ & 0,9 \\
\hline Диастолическая дисфункция 1-го типа & $134(97,8 \%)$ & $65(91,5 \%)$ & 0,007 \\
\hline Диастолическая дисфункция 2-го типа & $2(1,4)$ & $4(5,6 \%)$ & 0,009 \\
\hline Диастолическая дисфункция 3-го типа & $1(0,8 \%)$ & $2(2,8 \%)$ & 0,02 \\
\hline Систолическое давление в легочной артерии, мм рт. ст. & $37 \pm 6$ & $45 \pm 10$ & $<0,001$ \\
\hline
\end{tabular}

несмотря на незначительную площадь отверстия АК AVA $<1$ сM $^{2}$. Средний возраст $61 \pm 10$ лет, 13 пациентов женского пола.

Эхокардиграфия. Все пациенты проходили комплексную эхокардиографию с использованием ультразвуковых аппаратов (GE VIVID 7 PRO, VIVID 9, Норвегия). Трансторакальная эхокардиографическая оценка изучаемых параметров у пациентов проводилась в интервале частоты сердечных сокращений 60-80 уд/мин и после нормализации значений артериального давления (<150 и 90 мм рт. ст.). Эхокардиографические измерения выполняли в соответствии с рекомендациями Американского и Европейского обществ эхокардиографии [15]. Скорости и градиенты кровотока были получены из интегралов линейной скорости кровотока в соответствии с уравнением Бернулли. Измерение проводилось непрерывным волновым допплером на уровне АК в апикальной 5-камерной проекции. УО ЛЖ измеряли с помощью импульсного допплера в выходном тракте Лж и индексировали на площадь поверхности тела. Расчет AVA проводили с помощью уравнения непрерывности с выполнением всех условий для точного расчета показателей. Безразмерный индекс (DVI) рассчитан как отношение интеграла линейной скорости кровотока через выносящий тракт ЛЖ (опре- деленного с помощью импульсного допплера) к интегралу линейной скорости кровотока через АК (определенного непрерывно-волновым допплером). Конечно-диастолический объем (КДО) ЛЖ, конечносистолический объем (КСО) ЛЖ, ФВ ЛЖ, УО ЛЖ вычислялись по методу Симпсона (biplane): измерения производились в апикальных позициях, во взаимно перпендикулярных плоскостях, в 4- и 2-камерной проекциях. Измерение продольной деформации Лж выполнялось в автономном режиме методом спеклтрекинга. Показатель глобальной продольной деформации (GLS) Лж рассчитывали как среднее значение продольной деформации 2-, 3- и 4-камерного апикального обзора в состоянии покоя.

\section{Статистические методы}

База данных составлялась в виде электронных таблиц в программе Microsoft Office Excel 2007. Обработка данных производилась в демоверсии SPSS Statistics (Statistical Package for the Social Sciences). Все данные представлены как среднее \pm стандартное отклонение. Значимость различий между количественными признаками определялась при помощи t-критерия для независимых групп, между качественными признаками - при помощи непараметрических методов (критерий Манна-Уитни, $p<0,05)$. Категори- 


\begin{tabular}{|c|c|c|c|}
\hline Показатели & NFHG (n=137) & LFLG $(n=71)$ & $p$ \\
\hline Госпитальная летальность & $1(0,7 \%)$ & $3(4,2 \%)$ & 0,002 \\
\hline Атриовентрикулярные блокады 2-3-й степени & 0 & $3(4,2 \%)$ & $<0,001$ \\
\hline Послеоперационные пароксизмы ФП & $4(2,9 \%)$ & $8(11,2 \%)$ & $<0,001$ \\
\hline Полная блокада левой ножки пучка Гиса & $2(1,4 \%)$ & $6(8,4 \%)$ & $<0,001$ \\
\hline Постановка внутриаортального балонного контрапульсата & 0 & 0 & - \\
\hline Количество дней в отделении реанимации и интенсивной терапии & $5,8 \pm 2$ & $3,0 \pm 1$ & 0,008 \\
\hline
\end{tabular}

\begin{tabular}{|c|c|c|c|}
\hline Показатели & NFHG $(n=137)$ & LFLG $(n=71)$ & $p$ \\
\hline ФК 0 (NYHA) & $42(30,6 \%)$ & $5(7 \%)$ & $<0,001$ \\
\hline ФK I (NYHA) & $65(47,4 \%)$ & $20(28,2 \%)$ & $<0,001$ \\
\hline ФК II (NYHA) & $28(20,4 \%)$ & $40(56,3 \%)$ & $<0,001$ \\
\hline ФK III (NYHA) & $2(1,4 \%)$ & $6(8 \%)$ & $<0,001$ \\
\hline ФK IV (NYHA) & 0 & 0 & \\
\hline Средний ФК (NYHA) & $1,1 \pm 0,4$ & $1,5 \pm 0,6$ & 0,02 \\
\hline 6-минутный тест, м & $415 \pm 70$ & $380 \pm 84$ & $<0,001$ \\
\hline КДО лЖ, мл & $117 \pm 18$ & $94 \pm 27$ & $<0,001$ \\
\hline ФВ лЖ,\% & $65 \pm 3$ & $69 \pm 3$ & 0,03 \\
\hline Эффективный индекс УО ЛЖ, мл/м² & $37,6 \pm 3$ & $29 \pm 5$ & $<0,001$ \\
\hline GLS лж, \% & $16,8 \pm 3,3$ & $13,3 \pm 3$ & $<0,001$ \\
\hline Индекс объема лП & $40 \pm 4$ & $46 \pm 6$ & $<0,001$ \\
\hline Индекс массы ЛЖ, г/м² & $106 \pm 16$ & $133 \pm 29$ & $<0,001$ \\
\hline Gmax, мм рт. ст. & $13 \pm 4$ & $12,5 \pm 5$ & 0,09 \\
\hline Gmean, мм рт. ст. & $6,2 \pm 1,8$ & $6,0 \pm 1,6$ & 0,1 \\
\hline AVA, $\mathrm{CM}^{2}$ & $2,9 \pm 0,4$ & $2,8 \pm 0,2$ & 0,1 \\
\hline iAVA, $\mathrm{CM}^{2} / \mathrm{M}^{2}$ & $1,4 \pm 0,1$ & $1,4 \pm 0,1$ & 0,1 \\
\hline DVI & $0,65 \pm 0,1$ & $0,62 \pm 0,1$ & 0,1 \\
\hline Диастолическая дисфункция 1-го типа & $128(93,4 \%)$ & $65(91,5 \%)$ & 0,007 \\
\hline Диастолическая дисфункция 2-го типа & $2(1,4 \%)$ & $4(5,6 \%)$ & 0,009 \\
\hline Диастолическая дисфункция 3-го типа & $1(0,8 \%)$ & $2(2,8 \%)$ & 0,02 \\
\hline Систолическое давление в легочной артерии, мм рт. ст. & $27 \pm 6$ & $39 \pm 7$ & $<0,001$ \\
\hline
\end{tabular}

альные переменные выражались в процентах и сравнивались с использованием соответствующего критерия Пирсона $\chi^{2}$ или точного критерия Фишера. Статистически значимыми считали различия при уровне $p<0,05$. Модель множественной логистической регрессии использовалась для определения предикторов ранней смертности, а регрессия Кокса применялась для определения предикторов поздней смертности, включая все переменные, перечисленные в прилагаемых таблицах. Результаты выражены отношениями шансов и рисков, сообщенными с 95\% доверительными интервалами (Ди). Проведен анализ выживаемости по Каплану-Мейеру с построением кривых для групп, которые сравнивались с помощью логарифмической статистики. Первичной конечной точкой были 4-летняя смертность от всех причин и госпитализация по поводу хронической сердечной недостаточности $(\mathrm{CH})$.

\section{Результаты}

Предоперационные характеристики пациентов двух групп представлены в табл. 1. Максимальный период наблюдения обеих групп составил 50 мес, сред-

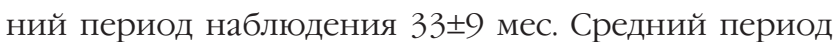
наблюдения групп пациентов с LFLG и с NFHG составил $33,7 \pm 8$ и 32,5 \pm 7 мес соответственно. Пациенты в группе c LFLG были чаще мужчинами, имели более высокую долю нарушения функции почек, артериальной гипертензии, нарушений ритма (фибрилляции предсердий - ФП), более низкую толерантность к физической нагрузке, худшую систолическую функцию ЛЖ и индекс УО $(p<0,05)$. Не было различий по времени ишемии и по времени искусственного кровообращения $(p \geqslant 0,05)$; см. табл. 1 .

Госпитальная летальность после хирургической коррекции была достоверно выше у пациентов в группе с LFLG, чем у пациентов в группе с NFHG, и составила $3(4,2 \%)$ и $1(0,7 \%)$ пациент соответственно $(p=0,002)$. У пациентов C LFLG отмечались более длительное пребывание в отделении реанимации и интенсивной терапии, более частое возникновение послеоперационных пароксизмов ФП, возникновение атриовентрикулярных блокад 2-3-й степени, требующих постановки электрокардиостимулятора (табл. 2).

Выживаемость в максимальный период наблюдения после хирургической коррекции АК для паци- 


\begin{tabular}{|c|c|c|c|}
\hline Показатели & ош & 95\% ди & $p$ \\
\hline Индекс массы ЛЖ, г/м² & 1,6 & $0,9-26$ & 0,05 \\
\hline GLS, \% & 0,56 & $0,40-1,1$ & 0,006 \\
\hline ФK (NYHA) & 0,9 & $0,86-1,0$ & 0,2 \\
\hline Индекс массы тела & 0,7 & $0,9-1,0$ & 0,1 \\
\hline КдО лж, мл & 0,3 & $0,98-1,0$ & 0,9 \\
\hline Индекс УО ЛЖ & 0,8 & $0,47-1,1$ & 0,008 \\
\hline ФВ лЖ, \% & 0,9 & $0,86-1,0$ & 0,22 \\
\hline Возраст, лет & 1,1 & $0,9-1,4$ & 0,05 \\
\hline
\end{tabular}

Рис. 1. Кривая выживаемости Каплана-Мейера.

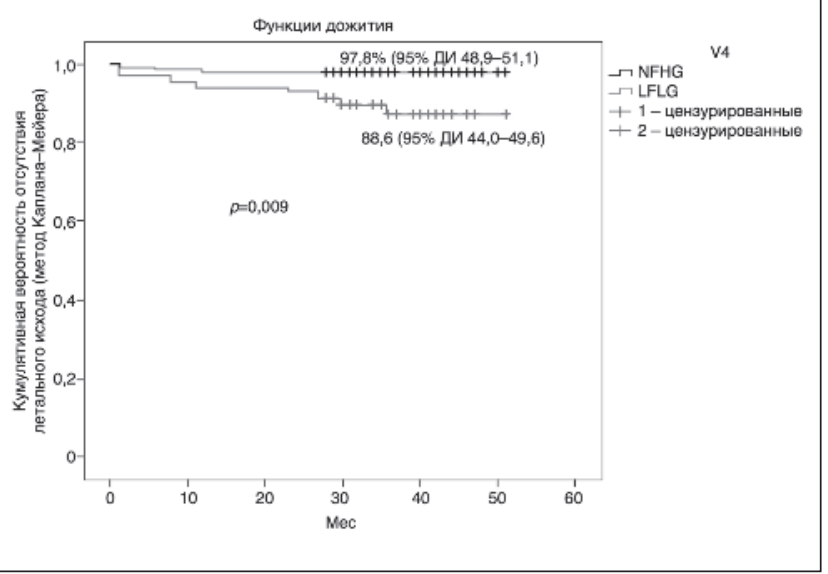

ентов с LFLG была значительно ниже, чем в группе пациентов с NFHG, и составила 88,6 (95\% ДИ 44-49,6) и 97,8 (95\% ДИ 48,9-51) соответственно ( $p=0,009)$; рис. 1 .

Нужно отметить, что в послеоперационном максимальном периоде наблюдения отмечались значительные улучшения в показателях трансаортальных градиентов, площади отверстия АК и не было никаких различий между группами по сократительной способности Лж. В обеих группах отмечалось значительное улучшение толерантности к физической нагрузке (см. табл. 2). Отмечалось улучшение ФК NYHA по сравнению с предоперационным пе-

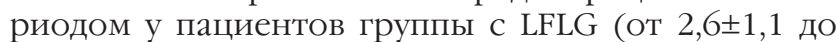
$1,5 \pm 0,6 ; p<0,001)$ и у пациентов группы с NFHG (c $2,0 \pm 1,3$ до $1,1 \pm 0,4 ; p<0,001)$. Тем не менее пациенты группы C LFLG чаще имели постоянную или рецидивирующую СН после хирургической коррекции АС, с меньшей свободой от госпитализации по поводу $\mathrm{CH}$. Свобода от госпитализаций по поводу $\mathrm{CH}$ в группе с LFLG была значительно ниже по сравнению с пациентами в группе с NFHG 87,1 (95\% Ди 43,6-49) и 97,1 (95\% ДИ 47,6-49,8) соответственно ( $p=0,001)$; рис. 2 .

Послеоперационное ремоделирование Лж у пациентов в группе с LFLG было более медленным, чем у пациентов в группе с NFHG. Пациенты в группе с LFLG в послеоперационном периоде наблюдения имели значительно меньшую регрессию массы ЛЖ по сравнению с пациентами в группе с NFHG $130 \pm 19$ г/м² и $112 \pm 16$ г $/ \mathrm{M}^{2}$ соответственно $(p<0,001)$. Несмотря на отсутствие различий в показателях ФВ ЛЖ, в послеоперационном периоде отмечались значительные различия в GLS между двумя группами $-13,3 \pm 2 \%$ и $16,5 \pm 2 \%$ соответственно $(p<0,001)$; табл. 3. Нужно отметить, что у пациентов в группе с LFLG в отдаленном
Рис. 2. Кривая Каплана-Мейера свободы от госпитализаций по поводу ХCH.

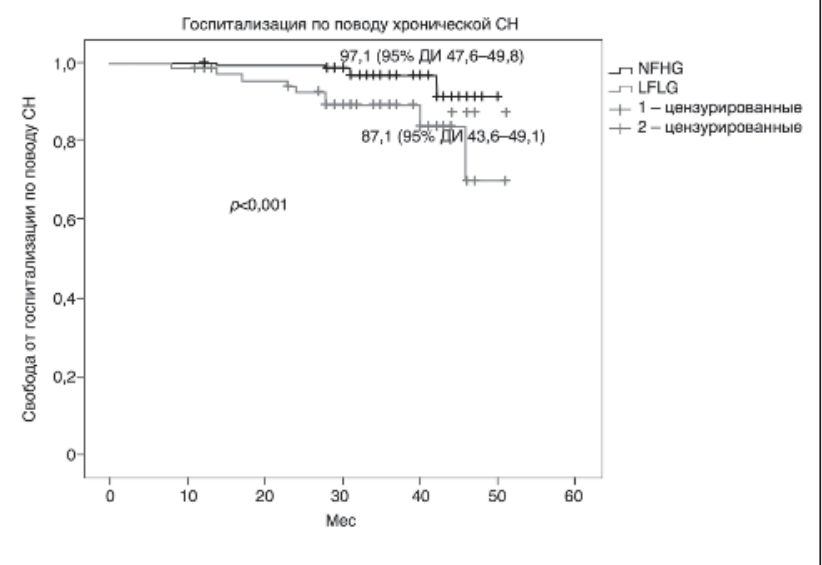

периоде наблюдения отмечались чаще нарушения ритма (ФП): LFLG - $4(5,6 \%)$ случая в группе, NFNG $1(0,7 \%)$ случай $(p=0,001) ;$ см. табл. 2.

По результатам мультивариантного логистического анализа значимыми независимыми предикторами госпитальной летальности у пациентов с LFLG являются индекс массы Лж [отношение шансов - ОШ $1,9$ (95\% ДИ 0,98-2,9); $p=0,01]$ и время ишемии [ОШ $1,7$ (95\% ДИ 0,96-2,4); $p=0,03]$. По результатам регрессии Кокса независимыми предикторами летальности в отдаленном периоде наблюдения у пациентов с LFLG являются индекс УО [ОШ 0,8 (95\% ДИ 0,9-1,1); $p=0,008]$ и GLS ЛЖ [ОШ 0,56 (95\% ДИ 0,47-1,1); $p=0,01]$; табл. 4 .

\section{Обсуждение}

Целью данного одноцентрового исследования являлось изучение влияния процедуры реконструкции створок АК аутоперикардом по методике Ozaki на группы пациентов с LFLG и NFHG. На сегодняшний день доказано, что пациенты с LFLG имеют более высокую смертность и более значительное количество неблагоприятных событий, чем пациенты c NFHG [3-8]. Одни исследователи связывают это с более высокими рисками пациент-протезного несоответствия, другие - с более выраженным нарушением геометрии и функции миокарда [9-13]. С другой стороны, очевидно, что прогноз LFLG с AC при медикаментозном лечении более неблагоприятный по сравнению с хирургическим лечением $[3,16,17]$. Учитывая тот факт, что пациенты с LFLG являются более тяжелыми в клиническом плане по сравнению с пациентами с NFHG и более чувствительны к остаточным градиентам на АК, становится очевидно, что для этой категории пациентов имплантация протезов с гемодинамикой, близкой к 
нативному клапану, может благоприятнее отразиться на отдаленных результатах. Одним из таких хирургических методов является реконструкция створок АК аутоперикардом по методике Ozaki. Метод реконструкции створок аутоперикардом обладает лучшей гемодинамикой АК по сравнению с биологическими протезами и не ограничивает пациента от осложнений, связанных с пациент-протезным несоответствием [14]. Учитывая столь значимые различия в гемодинамических характеристиках на АК по сравнению с биологическими протезами, мы предположили, что результаты после процедуры Ozaki могут несколько отличаться от известных на сегодняшний день исследований.

Предоперационные характеристики двух групп показали, что пациенты группы с LFLG по сравнению с пациентами группы c NFHG имели больше случаев гипертонической болезни в анамнезе, чаще имели патологию почек, более выраженную гипертрофию ЛЖ, дилатацию левого предсердия (ЛП), более выраженные явления легочной гипертензии и более низкие показатели контрактильной функции ЛЖ. По возрастным показателям отличий не было, однако в группе с LFLG было больше пациентов женского пола. У этой группы пациентов чаще отмечались признаки выраженной диастолической дисфункции Лж (см. табл. 1). Возможно, это и явилось причиной несколько более большего количества летальных событий и нарушения ритма на госпитальном этапе (см. табл. 2).

После проведенного оперативного лечения в обеих группах пациентов отмечались значительное улучшение симптомов, улучшение в показателях трансаортальных градиентов, регрессия гипертрофии ЛЖ, улучшение толерантности к физической нагрузке и улучшение ФК СН. В обеих группах не выявлено ни одного случая пациент-протезного несоответствия (см. табл. 3).

Однако, несмотря на положительное влияние хирургической коррекции АК в обеих группах, отдаленная летальность и количество госпитализаций, связанных с явлениями $\mathrm{CH}$, были достоверно выше в группе с LFLG. Свобода дожития в группе с LFLG и группе с NFHG составила 88,6 (95\% ДИ 44-49,6) и 97,8 (95\% ДИ 48,9-51) соответственно ( $p=0,009)$, свобода от госпитализации по поводу СН в группах составила 87,1 (95\% ДИ 43,6-49) и 97,1 (95\% ДИ 47,6-49,8) соответственно ( $p=0,001)$; см. рис. 2. По мнению некоторых авторов, это может быть связано с тем, что пациенты группы с LFLG имеют более позднюю стадию заболевания, что отражается более серьезным внутренним повреждением миокарда (больший фиброз миокарда) и худшей продольной функцией ЛЖ, более выраженной гипертрофией ЛЖ и наличием явлений, ограничивающих физиологическую работу ЛЖ. Это, в свою очередь, провоцирует низкий сердечный выброс, несмотря на наличие у некоторых пациентов сохраненной ФВ ЛЖ. Во многом данная группа пациентов сильно напоминает пациентов с СН с сохранной ФВ, для которых характерен неблагоприятный прогноз в отдаленном периоде [18-24].

Независимыми предикторами госпитальной летальности у пациентов с LFLG являются индекс массы лж [ОШ 1,9 (95\% Ди 0,98-2,9); $p=0,01]$ и время ишемии [ОШ 1,7 (95\% ДИ 0,96-2,4); $p=0,03$ ]. Во многом это объясняется тем, что протезирование створок аутоперикардом является достаточно трудоемким процессом, что, несомненно, требует длительного времени пережатия аорты, и пациенты с большой массой ЛЖ в значительной степени более подвержены неблагоприятным событиям.

Предикторами отдаленной летальности в группе пациентов C LFLG по регрессии Кокса являются индекс УО ЛЖ [ОШ 0,8 (95\% ДИ 0,9-1,1); $p=0,008]$ и GLS [ОШ 0,56 (95\% ДИ 0,47-1,1); $p=0,001]$.

По нашему мнению, логично, что индекс УО ЛЖ является значимым и независимым фактором выживаемости в отдаленном периоде, так как фактически он отражает изменение функции ЛЖ в результате нарушения как систолической, так и диастолической дисфункции ЛЖ и имеет доказанную сильную и независимую корреляцию с худшим исходом и выживаемостью. B исследовании D. Mohty и соавт. сообщалось, что показатель индекса УО Лж был независимым предиктором неблагоприятных событий в отдаленном периоде у пациентов с LFLG, что согласуется с результатами нашего исследования [25].

Другим не менее важным предиктором отдаленной летальности является показатель GLS. B нескольких крупных исследованиях сообщалось, что GLS значимый фактор риска летальных событий у пациентов с LFLG в отдаленном периоде наблюдения [26, 27]. Показатель GLS ЛЖ может быть более чувствительным маркером к изменениям систолической функции ЛЖ, чем ФВ ЛЖ у пациентов с LFLG. ФВ ЛЖ может длительное время недооценивать выраженность систолической дисфункции ЛЖ у пациентов с выраженным стенозом АК, особенно на фоне незначительных размеров ЛЖ и выраженной концентрической гипертрофии, несмотря на развитие субклинической систолической дисфункции миокарда ЛЖ [28, 29].

В нашем исследовании продемонстрировано, что пациенты группы с LFLG имеют несколько более высокую смертность и неблагоприятные события как на госпитальном этапе, так и в отдаленном периоде по сравнению с пациентами группы с NFHG. Во многом это связано с особенностями данной группы, которые обсуждались выше. Тем не менее очевидно, что пациенты обеих групп несомненно имеют значительную пользу от хирургической коррекции. Кроме этого, при сопоставлении результатов выживаемости нашего исследования с ранее проведенными после протезирования нам кажется, что пациенты группы с LFLG после процедуры Ozaki имеют несколько лучшие результаты. Несомненно, это требует дальнейшего изучения для того, чтобы понять, от какого из методов хирургической коррекции группа пациентов с LFLG имеет максимальную пользу.

\section{Выводы}

1. После процедуры Ozaki пациенты группы c LFLG имеют более высокие риски неблагоприятных событий как на госпитальном этапе, так и в отдаленном периоде наблюдения по сравнению с пациентами с NFHG.

2. Предикторами госпитальной летальности в группе пациентов с LFLG являются длительность ишемии и масса ЛЖ.

3. Предикторы летальности в отдаленном периоде у пациентов с LFLG - индекс УO ЛЖ и GLS ЛЖ.

Конфбликт интересов. Авторы заявляют об отсутствии конфликта интересов.

Conflict of interests. The authors declare that there is not conflict of interests. 


\section{Литература/References}

1. Nishimura RA, Otto CM, Bonow RO et al.2014 AHA/ACC guideline for the management of patients with valvular beart disease.J Am Coll Cardiol 2014; 63: e57-e185

2. Baumgartner H, Falk V, Bax JJ et al. 2017 ESC/EACTS Guidelines for the Management of Valvular Heart Disease: The Task Force for the Management of Valvular Heart Disease of the European Society of Cardiology (ESC) and the European Association for Cardio-Thoracic Surgery (EACTS). Eur Heart J 2017; Aug 26 [Epub abead of print].

3. Clavel MA, Berthelot-Richer $M$, Le Ven $F$ et al. Impact of classic and paradoxical low flow on survival after aortic valve replacement for severe aortic stenosis. J Am Coll Cardiol 2015; 65: 645-53.

4. Levy F, Laurent M, Monin JL et al. Aortic valve replacement for low-flow/low-gradient aortic stenosis. Operative risk stratification and long-term outcome: a European multicenter study. J Am Coll Cardiol 2008; 51:1466-72.

5. Tribouilloy C, Levy F, Rusinaru D et al. Outcome after aortic valve replacement for low-flow/low-gradient aortic stenosis without contractile reserve on dobutamine stress echocardiography.J Am Coll Cardiol 2009; 53: 1865-73.

6. Connolly HM, Ob JK, Schaff HV et al. Severe aortic stenosis with low transvalvular gradient and severe left ventricular dysfunction: result of aortic valve replacement in 52 patients. Circulation 2000; 101: 1940-6.

7. Mohty D, Magne J, Deltreuil $M$ et al. Outcome and impact of surgery in paradoxical low-flow, low-gradient severe aortic stenosis and preserved left ventricular ejection fraction: a cardiac catheterization study. Circulation 2013; 128: S235-S242. bttps://doi.org/10.1161/CIRCULATIONAHA. 112.000031

8. Lopez-Marco A, Miller H, Youbana A et al. Low-flow low-gradient aortic stenosis: surgical outcomes and mid-term results after isolated aortic valve replacement. Eur J Cardio Thor Surg 2016; 49 (6): 1685-90. bttps://doi.org/10.1093/ ejcts/ezv449

9. O'Sullivan CJ, Englberger L, Hosek $N$ et al. Clinical outcomes and revascularization strategies in patients with low-flow, low-gradient severe aortic valve stenosis according to the assigned treatment modality. J Am Coll Cardiol 2015; 8: 70417.

10. Brogan W, Graybursn P, Lange R, Hills I. Prognosis after valve replacement in patients with severe aortic stenosis and low transvalvular pressure gradient. J Am Coll Cardiol 1993; 21 : 1657-60.

11. Pereira JJ, Lauer MS, Bashir M et al. Survival after aortic valve replacement for severe aortic stenosis with low transvalvular gradients and severe left ventricular dysfunction. J Am Coll Cardiol 2002; 39: 1356-63.

12. Blitz L, Gorman M, Hermann H. Results of aortic valve replacement for aortic stenosis with relatively low transvavluar pressure gradients. Am J Cardiol 1998; 81: 358-62.

13. Pibarot P, Dumesnil JG. Valve prosthesis-patient mismatch, 1978 to 2011: from original concept to compelling evidence.J Am Coll Cardiol 2012; 60: 1136-9.

14. Базылев В.В., Россейкин Е.В., Бабуков Р.М. и др. Сравнение ближайиих и среднесрочных результатов у пациентов с узким корнем аорты после протезирования аотрального клапана биологическим протезом с аннулорасииряюшей пластикой корня аорты и протезированием створок аутоперикардом по методике ОZАКI. Клин. и эксперим. хирургия. 2019; 7 (1): 34-43.

[Bazylev V.V., Rosseikin E.V., Babukov R.M. et al. Sravnenie blizhaishikh i srednesrochnykh rezul'tatov u patsientov s uzkim kornem aorty posle protezirovaniia aotral'nogo klapana bio- logicheskim protezom s annulorasshiriaiushchei plastikoi kornia aorty $i$ protezirovaniem stvorok autoperikardom po metodike OZAKI. Klin. i eksperim. khirurgiia. 2019; 7 (1): 3443 (in Russian).]

15. Baumgartner H Chair et al. Recommendations for echocardiography Assessment of aortic valve stenosis: focused European Association Update Cardiovascular Tomography and American Societyechocardiography. J Am Soc Echocardiogr 2017; 30: 372-92

16. Awtry E, Davidoff R. Low-flow/low-gradient aortic stenosis. Circulation 2011; 124: 739-41.

17. Pibarot P, Dumesnil JG. Low-flow low-gradient aortic stenosis with normal and depressed left ventricular ejection fraction. J Am Coll Cardiol 2012; 60: 1845-53.

18. Barasch E, Fan D, Chukwu EO et al. Severe isolated aortic stenosis with normal left ventricular systolic function and low transvalvular gradients: pathophysiologic and prognostic insights. J Heart Valve Dis 2008; 17: 81-8.

19. Lancellotti P, Donal E, Magne J et al. Impact of global left ventricular afterload on left ventricular function in asymptomatic severe aortic stenosis: a two-dimensional speckle-tracking study. Eur J Echocardiogr 2010; 11: 537-43.

20. Herrmann S, Stork S, Niemann $M$ et al. Low-gradient aortic valve stenosis: Myocardial fibrosis and its influence on function and outcome.J Am Coll Cardiol 2011; 58: 402-12.

21. Cramariuc D, Cioffi G, Rieck AE et al. Low-flow aortic stenosis in asymptomatic patients: valvular arterial impedance and systolic function from the SEAS substudy. J Am Coll Cardiol 2009; p. 390-9.

22. Pai RG, Varadarajan P, Razzouk A. Survival benefit of aortic valve replacement in patients with severe aortic stenosis with low ejection fraction and low gradient with normal ejection fraction. Ann Thorac Surg 2008; 86: 1781-9.

23. Lee SP, Kim YJ, Kim JH et al. Deterioration of myocardial function in paradoxical low-flow severe aortic stenosis: two-dimensional strain analysis. J Am Soc Echocardiogr 2011; 24: 976-83.

24. Adda J, Mielot C, Giorgi R et al. Low-flow, low-gradient severe aortic stenosis despite normal ejection fraction is associated with severe left ventricular dysfunction as assessed by speckletracking echocardiography: a multicenter study. Circ Cardiovasc Imaging 2012; 5: 27-35.

25. Mohty D, Boulogne C, Magne J et al. Prevalence and long-term outcome of aortic prosthesis-patient mismatch in patients with paradoxical low-flow severe aortic stenosis. Circulation 2014; 130 (11; Suppl. 1): S25-31.

26. D'Andrea A, Carbone A, Agricola E et al. Predictive Value of Left Ventricular Myocardial Deformation for Left Ventricular Remodeling in Patients With Classical Low-Flow, Low-Gradient Aortic Stenosis Undergoing Transcatheter Aortic Valve Replacement. J Am Soc Echocardiogr 2019; 32 (6): 730-6. DOI: 10.1016/j.echo.2019.03.002

27. Dabou A, Bartko PE, Capoulade R et al. Usefulness of global left ventricular longitudinal strain for risk stratification in low ejection fraction, low-gradient aortic stenosis: results from the multicenter True or Pseudo-Severe Aortic Stenosis study. Circ Cardiovasc Imaging 2015; 8 (3): e002117. DOI: 10.1161/CIRCIMAGING.114.002117

28. Lancellotti P, Donal E, Magne J et al. Risk stratification in asymptomatic moderate to severe aortic stenosis: the importance of the valvular, arterial and ventricular interplay. Heart 2010; 96: 1364-71.DOI: 10.1136/ hrt.2009.190942

29. Delgado V, Tops LF, van Bommel RJ et al. Strain analysis in patients with severe aortic stenosis and preserved left ventricular ejection fraction undergoing surgical valve replacement. Eur Heart J 2009; 30: 3037-47. DOI: 10.1093/eurbeartj/ehp351 


\section{Информация об авторах / Information about the authors}

Базылев Владлен Владленович - д-р мед. наук, проф., глав. врач ФГБУ ФЦССХ. E-mail: cardio-penza@yandex.ru

Тунгусов Дмитрий Сергеевич - канд. мед. наук, сердечно-сосудистый хирург, зав. 2-м КХО ФГБУ ФЦССХ

Бабуков Руслан Медарисович - врач-кардиолог, врач ультразвуковой диагностики ФГБУ ФЦССХ

Бартош Федор Леонидович - врач ультразвуковой диагностики, врач-кардиолог ФГБУ ФЦССХ

Микуляк Артур Иванович - сердечно-сосудистый хирург ФГБУ ФЦССХ

Горшкова Алена Витальевна - врач ультразвуковой диагностики ФГБУ ФЦССХ
Vladlen V. Bazylev - D. Sci. (Med.), Prof., Federal Center of Cardiovascular Surgery. E-mail: cardio-penza@yandex.ru

Dmitrii S. Tungusov - Cand. Sci. (Med.), Federal Center of Cardiovascular Surgery

Ruslan M. Babukov - cardiologist, Federal Center of Cardiovascular Surgery

Fedor L. Bartosh - cardiologist, Federal Center of Cardiovascular Surgery

Artur I. Mikulyak - cardiovascular surgeon, Federal Center of Cardiovascular Surgery

Alena V. Gorshkova - ultrasound doctor, Federal Center of Cardiovascular Surgery

Статья поступила в редакцию / The article received: 15.04.2020

Статья принята к печати / The article approved for publication: 24.07.2020 